\section{Potential role of systemic thrombolysis in acute submassive intermediate risk pulmonary embolism: review and future perspectives}

\author{
Mohamed Teleb, Mateo Porres-Aguilar, Javier E. Anaya-Ayala, Carlos Rodriguez-Castro, \\ Mateo Porres-Muñoz and Debabrata Mukherjee
}

\begin{abstract}
Submassive (intermediate risk) pulmonary embolism (PE) continues to be a significantly morbid disease process that remains unrecognized, inadequately risk stratified and suboptimally treated. Appropriate early clinical and imaging-based risk stratification represents the cornerstone for adequate therapeutic decision making, particularly for the selection of candidates who may benefit the most from systemic thrombolysis. The relevance of estimating clinical prognostic scores, in combination with imaging data, for accurate assessment of right ventricular function and laboratory biomarkers, indicative of myocardial injury for identification of normotensive patients at intermediate risk for an adverse shortterm outcome are emphasized in this review. Recent clinical trials have demonstrated improvement in hemodynamics and mortality with the use of systemic thrombolysis among intermediate risk patients; however, it came at the cost of a significantly increased risk of major bleeding. Catheter-based therapies have garnered considerable clinical interest in recent years; of particular note is the ultrasound accelerated catheter-directed thrombolysis which has emerged as a novel and attractive alternative therapeutic modality with an increasing number of single center studies and ongoing randomized trials. Our review focuses on the major trials and studies involved in submassive PE in the recent literature including the role of thrombolytic therapy. We include major trials with reasonable sample size and extensive review of the potential side effects, such as major bleeding.
\end{abstract}

Keywords: clinical outcomes, right ventricular dysfunction, risk stratification, submassive pulmonary embolism, systemic thrombolysis

\section{Introduction}

Acute massive pulmonary embolism (PE) is one of the most frequent and lethal cardiovascular events, associated with an almost $95 \%$ mortality rate. This clinical presentation requires an aggressive therapeutic and cardiopulmonary resuscitative strategy such as systemic thrombolysis, catheter-based therapies or surgical embolectomy [Goldhaber et al. 1999; Jaff et al. 2011]. Studies have demonstrated that acute PE induced right ventricular (RV) failure is the most common cause of a 30-day mortality rate [Jaff et al. 2011; Wood, 2002]. The hemodynamic status is the best prognostic factor to determine implication for short-term mortality in acute PE patients. Massive PE is characterized by persistent arterial hypotension and/or cardiogenic shock with an increased short-term mortality rate [Wood, 2002]. Table 1 lists shows specific echocardiographic objective signs of RV dysfunction.

Acute submassive PE is defined by the presence of normotensive patients with signs of RV dysfunction and/or myocardial injury with a short-term mortality rate ranging from $3 \%$ to $15 \%$. Table 2 shows our proposed definition criteria of acute severe submassive $\mathrm{PE}$; the best therapeutic approach for submassive PE remains controversial
Ther Adv Cardiovasc Dis 2016, Vol. 10(2) 103-110 DOI: $10.1177 /$ 1753944716630694

(c) The Author(s), 2016 Reprints and permissions: http://www.sagepub.co.uk/ journalsPermissions.nav
Correspondence to: Mohamed Teleb, MD Texas Tech Health University Health Sciences Center, Department of Internal Medicine, 4800 Alberta Avenue, El Paso, TX 79905, USA Mohamed.telebattuhsc. edu

Mateo Porres-Aguilar, MD Carlos Rodriguez-Castro, MD

Department of Internal Medicine, Texas Tech University Health Sciences Center, El Paso, TX, USA

Javier E. Anaya-Ayala, MD Department of Surgery, Section of Vascular

Surgery and Endovascular Therapy, Instituto Nacional de Ciencias Médicas y Nutricion 'Salvador Zubirán', Mexico City, Mexico

Mateo Porres-Muñoz, MD Academic Secretary, Universidad Autonoma de Tamaulipas School of

Medicine, Department of Internal Medicine, Beneficencia Española de Tampico, Tampico, Mexico Debabrata Mukherjee, MD Division of Cardiovascular Diseases, Texas Tech University Health Sciences Center, El Paso, Texas, USA 
Table 1. Echocardiographic criteria for RV dysfunction.

\begin{tabular}{|c|c|c|}
\hline \multicolumn{3}{|c|}{ Qualitative criteria: RV hypokinesis (mild, moderate, severe) } \\
\hline Quantitative criteria & RV dilation & Pulmonary hypertension \\
\hline & $\begin{array}{l}\text { RV:LV end diastolic diameter } \\
>1 \mathrm{RV} \text { end-diastolic diameter } \\
>30 \mathrm{~mm}\end{array}$ & $\begin{array}{l}\text { Pulmonary artery systolic pressure }>30 \\
\mathrm{mmHg} \text {. } \\
\text { Tricuspid regurgitant velocity }>2.8 \mathrm{~m} / \mathrm{s} \\
\text { Pulmonary artery mean pressure }>20 \\
\mathrm{mmHg}\end{array}$ \\
\hline
\end{tabular}

Table 2. Proposed diagnostic definition of severe acute submassive pulmonary embolism.

\begin{tabular}{|c|c|c|c|c|}
\hline Definition & $\begin{array}{l}\text { Echocardiographic } \\
\text { findings }\end{array}$ & BNP & EKG & $\begin{array}{l}\text { Elevated } \\
\text { troponin I }\end{array}$ \\
\hline $\begin{array}{l}\text { Systolic blood } \\
\text { pressure }>90 \\
\text { mmHg and RV } \\
\text { dysfunction } \\
\text { or myocardial } \\
\text { necrosis } \\
\text { defined by one } \\
\text { of the following. }\end{array}$ & $\begin{array}{l}\text { RV dilation lapical } \\
\text { 4-chamber RV } \\
\text { diameter divided by LV } \\
\text { diameter }>0.9 \text { ) or RV } \\
\text { systolic dysfunction on } \\
\text { echocardiography or RV } \\
\text { dilation (4-chamber RV } \\
\text { diameter divided by LV } \\
\text { diameter }>0.9 \text { ) on } \mathrm{CT}\end{array}$ & $\begin{array}{l}\text { Elevation of BNP } \\
\text { (>90 pg/ml), or } N- \\
\text { terminal pro-BNP } \\
\text { (>500 pg/ml) }\end{array}$ & $\begin{array}{l}\text { EKG changes } \\
\text { Inew complete } \\
\text { or incomplete } \\
\text { right bundle } \\
\text { branch block, } \\
\text { anteroseptal } \\
\text { ST elevation or } \\
\text { depression, or } \\
\text { anteroseptal T } \\
\text { wave inversion }\end{array}$ & $\begin{array}{l}\text { Troponin I } \\
\text { (>0.4 ng/ml) } \\
\text { or troponin T } \\
\text { (>0.1 ng/ml) }\end{array}$ \\
\hline
\end{tabular}

[Ince et al. 2014; Porres-Aguilar and PorresMuñoz, 2014]. Patients presenting as hemodynamically stable could have a similar short-term mortality risk as those with massive PE if they are appropriately assessed as clinically risk stratified early on (Table 3), eventually leading to more applicable and aggressive therapies [Kucher et al. 2005; Sanchez et al. 2008; Schoepf et al. 2004; Becattini and Agnelli, 2008]. Although systemic thrombolysis has demonstrated hemodynamic and angiographic improvements compared with standard intravenous (IV) heparin in patients with massive PE, studies have not provided clear evidence in regard to the clinical outcomes such as morbidity and mortality; however, there has been a noteworthy trend towards reduction in PE-related mortality and recurrent PE episodes [Kucher et al. 2006; Jerjes-Sanchez et al. 1995; Stein and Matta, 2012; Wan et al. 2003]. Figure 1 shows an integrated multidisciplinary therapeutic approach in sub massive PE.

Clinical investigations have explored the potential role of systemic thrombolysis in acute severe submassive PE following an adequate integrated risk-stratification (Figure 2). The common risk stratification approach includes evidence of objective RV dysfunction detected by transthoracic echocardiography (TTE), chest computed tomographic angiography (CTA), elevated cardiac injury biomarkers such as troponin-I, brain natriuretic peptide (BNP) and pro-BNP. An additional approach includes cardiopulmonary clinical status such as moderate or severe hypoxemia by oxygen saturation (O2Sat) and arterial partial pressure of oxygen $(\mathrm{PaO} 2)$ [Tapson, 2013; Jiménez, 2013; Lankeit and Konstantinides, 2012].

The question remains whether the indication of systemic thrombolysis could positively impact the clinical outcomes, e.g. survival, quality of life and the development of persistent pulmonary arterial hypertension (PAH) in the subgroup of patients with submassive PE. This review focuses on current evidence of clinical trials and the management of submassive PE; other possible treatment modalities such as catheter-based therapies using local thrombolysis or direct extraction/embolectomy have been excluded. 
Table 3. Pulmonary Embolism Severity Index.

\begin{tabular}{|c|c|}
\hline Prognostic variables & Points assigned \\
\hline \multicolumn{2}{|l|}{ Demographics } \\
\hline Age (years) & Age \\
\hline Male sex & +10 \\
\hline \multicolumn{2}{|l|}{ Comorbid conditions } \\
\hline Cancer & +30 \\
\hline Heart failure & +10 \\
\hline Chronic lung disease & +10 \\
\hline \multicolumn{2}{|l|}{ Critical findings } \\
\hline Pulse $>110$ beats per minute & +20 \\
\hline $\begin{array}{l}\text { Systolic blood pressure }<100 \\
\mathrm{~mm} \mathrm{Hg}\end{array}$ & +30 \\
\hline $\begin{array}{l}\text { Respiratory rate }>30 \text { breaths } \\
\text { per minute }\end{array}$ & +20 \\
\hline Temperature $<36^{\circ} \mathrm{C}$ & +20 \\
\hline Altered mental status ${ }^{*}$ & +60 \\
\hline $\begin{array}{l}\text { Arterial oxygen saturation } \\
<90 \% \$\end{array}$ & +20 \\
\hline \multicolumn{2}{|c|}{$\begin{array}{l}\text { Class I (very low risk: } 65 \text { points, mortality } 0-1.6 \% \text { ). } \\
\text { Class II (low risk: } 66-86 \text { points, mortality } 1.7-3.5 \% \text { ). } \\
\text { Class III (intermediate risk: } 86-105 \text { points, mortality } \\
3.2-7.1 \% \text { ). } \\
\text { Class IV (high risk: } 106-125 \text { points, mortality } 4-11.4 \%) \text {. } \\
\text { Class V (very high risk: }>125 \text { points, mortality } 10-24.5 \% \text { ). } \\
\text { *Disorientation, lethargy, stupor, or coma. } \\
\text { \$With or without supplementary oxygen. }\end{array}$} \\
\hline
\end{tabular}

\section{Current evidence of clinical trials evaluating systemic thrombolysis in acute submassive PE}

\section{Meta-analysis evaluation}

Chatterjee and colleagues published a meta-analysis consisting of 16 clinical trials including 2115 patients [Chatterjee et al. 2014]. These studies were randomized clinical trials comparing systemic thrombolytic therapy versus standard anticoagulation therapy. The results indicated a decreased all-cause mortality [odds ratio (OR), $0.53 ; 95 \%$ confidence interval (CI), 0.32-0.88; $2.17 \%$ versus $3.89 \%$ with anticoagulants; number needed to treat $(\mathrm{NNT})=59$ with absolute risk reduction of $1.12 \%]$. The risk of major bleeding was noted to be greater [OR, 2.73; 95\% CI, $1.91-3.91 ; 9.24 \%$ versus $3.42 \%$; number needed to harm $(\mathrm{NNH})=18$ ]. Thrombolysis was noted to have a lower risk of recurrent PE (OR, 0.40; $95 \%$ CI, $0.22-0.74 ; \quad 1.17 \%$ versus $3.04 \%$; NNT =54). A lower mortality rate was observed in submassive PE trials (OR, 0.48; 95\% CI, 0.250.92) [Lankeit and Konstantinides, 2012]. Nakamura and colleagues conducted a similar meta-analysis that included 6 randomized control trials consisting of 1510 patients; the results were

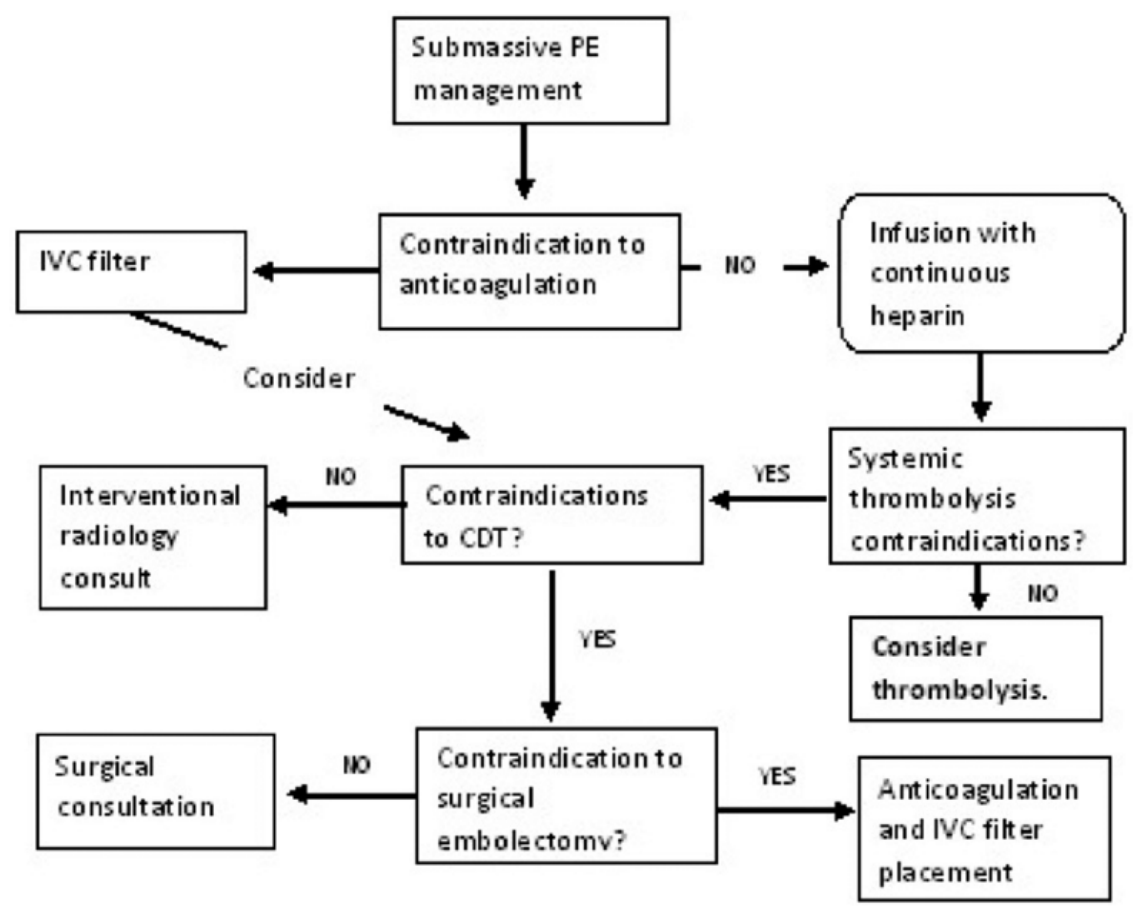

Figure 1. Proposed algorithm treatment for the management of submassive pulmonary embolism. CDT, catheter directed therapy; IVC, inferior vena cava. 


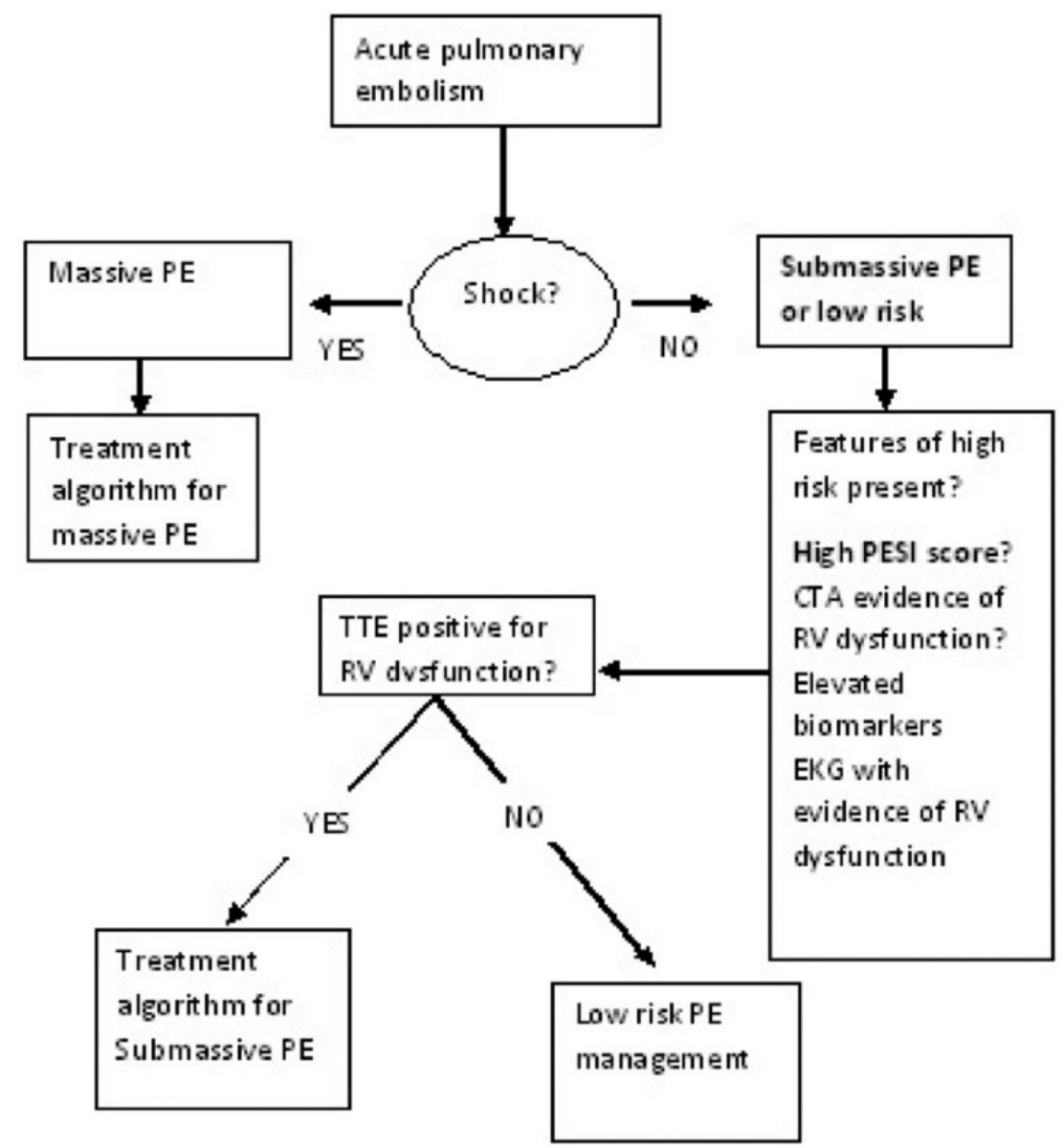

Figure 2. Proposed algorithm for risk stratification of acute pulmonary embolism. CTA, computed tomography angiography; EKG, electrocardiogram; PE, pulmonary embolism; PESI, pulmonary embolism severity index; RV, right ventricle; TTE, transthoracic echocardiogram.

similar to that of Chatterjee and colleagues [Chatterjee et al. 2014]. However, there was a slightly larger absolute risk difference (1.6\%), although this was not significant due to the smaller sample size [Nakamura et al. 2014].

\section{Management Strategies and Prognosis of Pulmonary Embolism Trial-3 (MAPPET-3) trial}

Konstantinides and colleagues evaluated a multicenter, randomized, double-blind, placebo controlled trial in Germany [Konstantinides et al. 2002]. It consisted of 256 patients with submassive $\mathrm{PE}$ and objective evidence of $\mathrm{PAH}$ or RV dysfunction without hypotension or shock. Patients received either IV heparin plus IV 100 $\mathrm{mg}$ of alteplase (118 patients) versus IV heparin plus placebo (138 patients) over a 2-hour time span. The primary endpoint was to evaluate inhospital death rates or clinical outcomes requiring an increased dosage of therapy. The secondary endpoint was to evaluate safety and tolerability. The incidence of the primary endpoint was higher among the heparin patients and placebo group $(p$ $=0.006)$. It was noted also that the 30 days' event-free survival rate was higher in the heparin plus alteplase group $(p=0.005)$. This difference was directly attributed to a higher incidence of increased therapy in the heparin plus placebo group $(24.6 \%$ versus $10.2 \%)$, with both groups evidencing low mortality rates $3.4 \%$ in the heparin plus alteplase group versus $2.2 \%$ in the heparin plus placebo group, $p=0.71$ ). A significant find, resulting from the research, was nonfatal or intracranial hemorrhage occurring in the heparin plus alteplase group versus one fatal bleeding episode in the heparin plus placebo group. The investigators concluded that alteplase plus heparin in submassive PE can improve clinical outcomes in otherwise stable patients; it can also prevent deteriorated clinical condition if escalation of treatment is needed. 
Tenecteplase Italian Pulmonary Embolism Study (TIPES)

This was a multi-center, randomized, doubleblind, placebo-controlled study where Becattini and colleagues evaluated the effect of IV tenecteplase on RV dysfunction as assessed by echocardiogram in hemodynamically stable patients with submassive $\mathrm{PE}$ as the primary endpoint [Becattini et al. 2010]. The study included a total of 58 patients; however, the echocardiogram was adequate for only 51 analyses. It was estimated that 23 patients were randomized to tenecteplase and 28 to placebo. Tenecteplase was given as a weightadjusted bolus at a dose ranging between 30 and $50 \mathrm{mg}$, and all patients in the study received unfractionated heparin. The reduction of the RV to $\mathrm{LV}$ end-diastolic dimension ratio at 24 hours was statistically significant $(0.31 \pm 0.08$ in the tenecteplase group versus $0.10 \pm 0.07$ in placebo, $p=0.04$ ). Adverse events were noted as follows: two nonfatal bleeding occasions occurred in the tenecteplase group (one intracranial) and one episode in the placebo group; one patient had recurrent $\mathrm{PE}$ in the tenecteplase group versus three patients in the placebo group. The study also evaluated the 30 days' mortality rate which included 1 patient in the placebo group who died of myocardial infarction (MI). No further recurrent venous thromboembolism event or death occurred until day 30 . The $\mathrm{NNH}$, related to clinical events at 30 days, was noted to be 58.5 specifically with regard to death as we calculated that value. The investigators concluded that IV bolus weight-adjusted tenecteplase was associated with improvement of RV dysfunction at 24 hours; whether this benefit is associated with improvement in survival without excessive bleeding events still remains to be defined.

\section{Moderate Pulmonary Embolism Treated with Thrombolysis Trial (MOPETT)}

The study reflected a prospective, randomized, controlled, single-center open approach headed by Sharifi and colleagues where the effect of a 'safer dose' of recombinant tissue plasminogen activator (rTPA) as the choice of thrombolytic agent in the reduction of pulmonary arterial pressure in 'moderate' PE was evaluated [Sharifi et al. 2013]. The investigators defined 'moderate' PE as the presence of classic features of acute PE plus chest CTA involvement of $>70 \%$ of thrombus in $>2$ lobar arteries. They also extended the definition to the involvement of the main right or left pulmonary arteries or by a ventilation/perfusion lung scan showing high probability $\mathrm{PE}$ in $\geqslant 2$ lobes. The primary endpoint of the study was to evaluate the development of PAH and its endpoint, and also to evaluate recurrent $\mathrm{PE}$ at 28 months. The secondary endpoint was to evaluate total mortality, hospital stay duration, bleeding, recurrent $\mathrm{PE}$ and mortality with recurrent $\mathrm{PE}$ combined factors. About 121 patients were randomized to either IV rTPA $(n=61)$ at $50 \mathrm{mg}$ in those weighing $>50 \mathrm{~kg}$ or $0.5 \mathrm{mg} / \mathrm{kg}$ if $<50 \mathrm{~kg}$ plus anticoagulation (unfractionated heparin or subcutaneous enoxaparin). The other arm of the study was to randomize patients to receive anticoagulation therapy (control group, $n=60$ ) over a period of 22 months. PAH developed in 9 of 58 patients $(16 \%)$ in the thrombolysis plus anticoagulation group compared with 32 of 57 patients $(57 \%)$ in the control group. The composite endpoint of PAH developed in 9 of 58 patients (16\%) in the thrombolysis plus anticoagulation group compared with 35 of 56 patients $(63 \%)$ in the control group. Secondary endpoint evaluation revealed the following. Hospital duration was 2.2 \pm 0.5 days in the thrombolysis plus anticoagulation group versus $4.9 \pm 0.8$ days in the control group; the combination between death and recurrent PE was $1(1.6 \%)$ in the thrombolysis plus anticoagulation group versus $6(10 \%)$ in the control group $(p=0.048)$; no bleeding episodes noted in all groups. Some variations noted in terms of NNT/NNH were 2.1 and 11.9 respectively. The investigators concluded that a 'safe dose' of rTPA was adequate and effective with a reduction in pulmonary arterial pressures as a possible alternative for the same dose when used in a catheter related thrombolysis approach.

\section{Pulmonary Embolism THrOmbolysis (PEITHO) trial}

In this prospective, multi-center, international, randomized, double-blind, placebo controlled study with the overall goal of comparing thrombolysis with IV Tenecteplase in normotensive patients with acute submassive $\mathrm{PE}$ versus placebo $(n=1006)$, all study subjects received anticoagulation [Meyer et al. 2014]. The primary endpoint was to evaluate all-cause mortality or hemodynamic collapse within 7 days of randomization. The secondary endpoint focused on assessing the safety of tenecteplase such as nonintracranial bleeding, strokes within 7 days and serious adverse events. Although the primary outcome of all-cause mortality or hemodynamic collapse occurred in 13 patients $(2.6 \%)$ randomized to the IV tenecteplase 
group compared with 28 patients (5.6\%) randomized to placebo $(p=0.015)$, there were more major nonintracranial bleeding events with 32 patients $(6.3 \%)$ in the IV tenecteplase arm compared with 6 patients $(1.5 \%)$ in the placebo arm. All strokes were noted to be higher in the $\mathrm{T}$ =tenecteplase arm (12 patients, $2.4 \%)$ versus placebo (1 patient, $0.2 \%$ ). In contrast to these findings, serious adverse events were lower in the tenecteplase arm (29 patients, 5.7\%) versus placebo (39 patients, 7.8\%). Another observed finding was the all-cause mortality within 30 days of randomization was lower in the tenecteplase arm (12 patients, 2.4\%) versus placebo (16 patients, $3.2 \%)$. The study also evaluated a specific subgroup defined by age $(<75$ years versus $>75$ years). $\mathrm{NNH}$ from these subgroups included major extracranial bleeding and was noted to be 38.5 in subgroup patients $<75$ years and 9.5 in those $>75$ years old. The authors concluded that, in patients with appropriate integrated prompt risk stratification for submassive (intermediate risk) acute PE, IV tenecteplase significantly reduced the primary endpoint of death or hemodynamic collapse within 7 days of randomization. They also confirmed that early systemic thrombolytic therapy prevents RV dysfunction and myocardial injury. The trial also addressed that secondary thrombolysis, after clinical deterioration, is very effective and likely the most reasonable approach in patients with submassive PE. One of the major negative points from this trial included the significant increased risk of major bleeding events, specifically intracranial bleeding in elderly patients ( $>75$ years old).

\section{Other studies}

Riera-Mestre and colleagues conducted a retrospective cohort study of 15,944 patients with symptomatic acute PE identified from the multi center, international, prospective, Registro InformatizadodelaEnfermedad TromboEmbólica (RIETE registry). The main goal was to evaluate the all-cause mortality during the first 3 months after the diagnosis of a PE. In the normotensive subgroup patients, analysis of propensity scorematched pairs $(n=217)$ showed statistical significance in terms of increased risk of death in the thrombolysis arm versus no thrombolysis arm (OR 2.32; 95\% CI, 1.15-4.68; $p=0.018$ ) [RieraMestre et al. 2012].

Goldhaber and colleagues evaluated 2454 patients registered from the International Cooperative
Pulmonary Embolism Registry (ICOPER). Intracranial hemorrhage occurred in 3\% of patients who received thrombolytic therapy versus $0.3 \%$ who did not. It was also estimated that the incidence of any major bleeding was $22 \%$ among the thrombolytic therapy group versus $9 \%$ in nonthrombolytic group [Goldhaber et al. 1999].

Weinberg and Jaff conducted a study to evaluate the role of thrombolysis in normotensive patients with sub massive PE and the effect on RV strain. All patients received low molecular weight heparin followed by randomization to either tenecteplase or placebo. The study, although terminated prematurely, revealed some interesting findings including three adverse events in the placebo arm and one fatal intracranial bleed in the tenecteplase arm. In conclusion, it was noted that $37 \%$ in the placebo arm and $15 \%$ in the tenecteplase arm had at least one adverse event $(p=0.017)$ [Weinberg and Jaff, 2013].

Gao and colleagues published a meta-analysis of 8 clinical randomized trials involving a total of 1755 patients with intermediate risk PE. Patients with thrombolytic therapy experienced a lower mortality rate than patients in the anticoagulation cohort [relative risk (RR), 0.52; 95\% CI, 0.28$0.97 ; 1.39 \%$ (12/866) versus $2.92 \%$ (26/889)]. Compared with anticoagulation, thrombolytic therapy was associated with a higher risk of major bleeding (RR, 3.35; 95\% CI, 2.77-4.84; 32.78\% versus $8.94 \%)$. Furthermore, thrombolytic therapy was associated with a lower incidence of recurrent PE [RR, 0.33; 95\% CI, 0.15-0.73; $0.73 \% ; 0.73 \%$ (6/826) versus $2.72 \%$ (23/846)]. The authors concluded that, compared with anticoagulation thrombolytic therapy in patients with intermediate-risk PE, there is a lower all-cause mortality and recurrent PE risk despite increased major and minor bleeding [Gao et al. 2015].

\section{Conclusions and future considerations}

Novel approaches for the treatment over the past decade for massive and submassive PE have raised new clinical questions, both interesting and controversial, on the clinical utility, role, applicability of such therapeutic modalities and the specific criteria among investigators. Submassive PE is usually presented in normotensive patients with RV dysfunction and elevated biomarkers of myocardial injury/stress, which prompts clinicians for an early and adequate diagnosis, risk stratification for appropriate therapeutic decision making 
Table 4. Predictors of major bleeding following thrombolysis in acute pulmonary embolism.

\begin{tabular}{l}
\hline Characteristics \\
\hline Male gender \\
Severe obesity \\
Hemodynamic instability \\
Current cancer \\
Metastatic cancer \\
Chemotherapy within 6 months \\
Hypertension \\
Diabetes mellitus \\
Creatinine $>1.5 \mathrm{mg} / \mathrm{dl}$ \\
Previous deep vein thrombosis or pulmonary \\
embolism
\end{tabular}

during the short-term course of these patients. Research remains limited in terms of high quality data evaluating systemic thrombolysis in this clinical purview.

Our review focused on the main clinical trials addressing these issues with a particular emphasis on symptom improvement and evaluating hemorrhagic risk. In analysis of the forementioned trials in this review, we can note that all-cause mortality was similar in the 2 metaanalyses $(2.17 \%$ in thrombolysis versus $3.89 \%$ nonthrombolysis therapy), although the risk of major bleeding was higher $(9.24 \%$ versus $3.42 \%)$. When comparing the all-cause mortality or recurrent PE between the MAPPET-3, TIPES, MOPETT and PEITHO trials; the MAPPET-3 trial had the highest rate $(3.4 \%)$ versus the TIPES trial, which had 1 death at 3 days and another at day 30 from MI, indicating the possibility of lower effectiveness of heparin plus alteplase therapy compared with tenecteplase plus heparin. Another interesting finding was different values of bleeding risk (intracranial or nonintracranial major bleeding) as Table 4 describes the major clinical predictors of bleedings post-thrombolysis. It was noted that PEITHO trial had the highest level of major non intracranial bleeding $(6.3 \%)$ versus no bleeding at all in the MOPETT and MAPPET-3 trials. In short, IV tenecteplase had a better outcome in submassive PE treatment, although there was an increased risk of bleeding.

With regard to future trials, advancements will only continue to improve our current knowledge and standard treatment for submassive PE; clinical studies and more consolidated data can provide additional information regarding that point.

Catheter-based, pharmacomechanical therapies have gained considerable interest lately. In particular, ultrasound-facilitated, catheter-directed thrombolysis has emerged as an attractive alternative therapeutic modality with an increasing number of single-center studies and ongoing randomized trials with promising preliminary results [Kucher et al. 2014; Piazza et al. 2015]. This is especially true among the subgroup of patients with very high risk of internal or intracranial bleeding and with absolute contraindications for systemic thrombolysis. Major trials are mentioned in the literature such as SEATTLE 2 and the use of EkoSonic system with promising results.

\section{Funding}

This research received no specific grant from any funding agency in the public, commercial, or notfor-profit sectors.

\section{Conflict of interest statement}

The authors declare no conflicts of interest in preparing this article.

\section{References}

Becattini, C. and Agnelli, G. (2008) Predictors of mortality from pulmonary embolism and their influence on clinical management. Thromb Haemost 100: 747-751.

Becattini, C., Agnelli, G., Salvi, A., Grifoni, S., Pancaldi, L., Enea, I. et al. (2010) Bolus tenecteplase for right ventricular dysfunction in haemodynamically stable patients with pulmonary embolism. Thromb Res 125: e82-e86.

Chatterjee, S., Chakraborty, A., Weinberg, I., Kadakia, M., Wilensky, R., Sardar, P. et al. (2014) Thrombolysis for pulmonary embolism and risk of all-cause mortality, major bleeding, and intracranial hemorrhage: a meta-analysis. $\mathcal{F} \mathrm{Am} \mathrm{Med} \mathrm{Assoc} \mathrm{311:}$ 2414-2121.

Gao, G., Yang, P., Liu, M., Ding, M., Liu, G., Tong, Y. et al. (2015) Thrombolysis for acute intermediaterisk pulmonary embolism: a meta-analysis. Thromb Res 136: 932-937.

Goldhaber, S., Visani, L. and De Rosa, M. (1999) Acute pulmonary embolism: clinical outcomes in the International Cooperative Pulmonary Embolism Registry (ICOPER). Lancet 353: 1386-1389.

Ince, O., Altintas, N., Findik, S. and Sariaydin, M. (2014) Risk stratification in submassive pulmonary 
embolism via alveolar-arterial oxygen gradient. Hippokratia 18: 333-339.

Jaff, M., McMurtry, M., Archer, S., Cushman, M., Goldenberg, N., Goldhaber, S. et al. (2011) Management of massive and submassive pulmonary embolism, iliofemoral deep vein thrombosis, and chronic thromboembolic pulmonary hypertension: a scientific statement from the American Heart Association. Circulation 123: 1788-1830.

Jerjes-Sanchez, C., Ramírez-Rivera, A., de Lourdes García, M., Arriaga-Nava, R., Valencia, S., RosadoBuzzo, A. et al. (1995) Streptokinase and heparin versus heparin alone in massive pulmonary embolism: a randomized controlled trial. $\mathcal{F}$ Thromb Thrombolysis 2: 227-229.

Jiménez, D. (2013) Point: Should systemic lytic therapy be used for submassive pulmonary embolism?: Yes. Chest 143: 296-299.

Konstantinides, S., Geibel, A., Heusel, G., Heinrich, F. and Kasper, W. (2002) Heparin plus alteplase compared with heparin alone in patients with submassive pulmonary embolism. N Engl f Med 347: 1143-1150.

Kucher, N., Boekstegers, P., Muller, O., Kuppatt, C., Beyer-Westendorf, J., Heitzer, T. et al. (2014) Randomized, controlled trial, of ultrasound assisted catheter directed thrombolysis for acute intermediaterisk pulmonary embolism. Circulation 129: 479-486.

Kucher, N., Rossi, E., De Rosa, M. and Goldhaber, S. (2005) Prognostic role of echocardiography among patients with acute pulmonary embolism and systolic arterial pressure $90 \mathrm{mmHg}$ or higher. Arch Intern Med 165: 1777-1781.

Kucher, N., Rossi, E., De Rossa, M. and Goldhaber, S. (2006) Massive pulmonary embolism. Circulation 113: 577-582.

Lankeit, M. and Konstantinides, S. (2012) Thrombolytic therapy for submassive pulmonary embolism. Best Pract Res Clin Haematol 25: 379-389.

Meyer, G., Vicaut, E., Danays, T., Agnelli, G., Becattini, C., Beyer-Westendorf, J. et al. (2014) Fibrinolysis for patients with intermediate-risk pulmonary embolism. N Engl F Med 370: 1402-1411.

Nakamura, S., Takano, H., Kubota, Y., Asai, K. and Shimizu, W. (2014) Impact of the efficacy of thrombolytic therapy on the mortality of patients with acute submassive pulmonary embolism: a metaanalysis. F Thromb Haemost 12: 1086-1095.
Piazza, G., Hohlfelder, B., Jaff, M., Ouriel, K., Engelhardt, T., Sterling, K. et al. (2015) A prospective, single arm, multicenter trial of ultrasound-facilitated, catheter-directed, low dose fibrinolysis for acute massive and submassive pulmonary embolism. FACC Cardiovasc Interv 8: 1382-1392.

Porres-Aguilar, M. and Porres-Muñoz, M. (2014) Controversial and future role of thrombolysis and endovascular therapies in submassive pulmonary embolism. Arch Cardiol Mex 80: 143-144.

Riera-Mestre, A., Jiménez, D., Muriel, A., Lobo, J., Moores, L., Yusen, R. et al. (2012) Thrombolytic therapy and outcome of patients with an acute symptomatic pulmonary embolism. F Thromb Haemost 10: 751-759.

Sanchez, O., Trinquart, L., Colombet, I., Durieux, P., Huisman, M., Chatellier, G. et al. (2008) Prognostic value of right ventricular dysfunction in patients with hemodynamically stable acute pulmonary embolism: a systematic review. Eur Heart f 29: 1569-1577.

Schoepf, U., Kucher, N., Kipfmueller, F., Quiroz, R., Costello, P. and Goldhaber, S. (2004) Right ventricular enlargement on chest computed tomography: a predictor of early death in acute pulmonary embolism. Circulation 110: 3276-3280.

Sharifi, M., Bay, C., Skrocki, L., Rahimi, F. and Mehdipour, M. (2013) Moderate pulmonary embolism treated with thrombolysis (from the MOPETT trial). Am f Cardiol 111: 273-277.

Stein, P. and Matta, F. (2012) Thrombolytic therapy in unstable patients with acute pulmonary embolism: saves lives but is underused. $\mathrm{Am} \mathcal{F} \mathrm{Med}$ 125: 465-470.

Tapson, V. (2013) Thrombolytic therapy for acute pulmonary embolism. Semin Thromb Haemost 39: 452-458.

Wan, S., Quinlan, D., Agnelli, G. and Eikelboom, J. (2004) Thrombolysis compared with heparin for the initial treatment of pulmonary embolism: a metaanalysis of randomized controlled trials. Circulation 110: 744-749.

Weinberg, I. and Jaff, M. (2013) Treating large pulmonary emboli: do the guidelines guide us? Curr Opin Pulm Med 19: 413-421.

Wood, K. (2002) Major pulmonary embolism: review of pathophysiologic approach to the golden hour of hemodynamically significant pulmonary embolism. Chest 121: 877-905. 